\title{
Is cooking at home associated with better diet quality or weight-loss intention?
}

\author{
Julia A Wolfson* and Sara N Bleich \\ Department of Health Policy and Management, Bloomberg School of Public Health, Johns Hopkins University, \\ 624 N. Broadway, Baltimore, MD 21205, USA
}

Submitted 23 November 2013: Final revision received 6 May 2014: Accepted 6 August 2014: First published online 17 November 2014

\begin{abstract}
Objective: To examine national patterns in cooking frequency and diet quality among adults in the USA, overall and by weight-loss intention.

Design: Analysis of cross-sectional $24 \mathrm{~h}$ dietary recall and interview data. Diet quality measures included total kilojoules per day, grams of fat, sugar and carbohydrates per day, fast-food meals per week, and frozen/pizza and ready-toeat meals consumed in the past $30 \mathrm{~d}$. Multivariable regression analysis was used to test associations between frequency of cooking dinner per week (low (0-1), medium (2-5) and high (6-7)), dietary outcomes and weight-loss intention.

Setting: The 2007-2010 National Health and Nutrition Examination Survey.

Subjects: Adults aged 20 years and over ( $n$ 9569).

Results: In 2007-2010, 8 \% of adults lived in households in which someone cooked dinner 0-1 times/week and consumed, on an average day, 9627 total kilojoules, $86 \mathrm{~g}$ fat and $135 \mathrm{~g}$ sugar. Overall, compared with low cookers ( $0-1$ times/week), a high frequency of cooking dinner (6-7 times/week) was associated with lower consumption of daily kilojoules $(9054 v .9627 \mathrm{~kJ}, P=0.002)$, fat (81 v. $86 \mathrm{~g}$, $P=0.016)$ and sugar (119 v. $135 \mathrm{~g}, P<0 \cdot 001)$. Individuals trying to lose weight consumed fewer kilojoules than those not trying to lose weight, regardless of household cooking frequency (2111 v. $2281 \mathrm{~kJ} / \mathrm{d}, P<0 \cdot 006)$.

Conclusions: Cooking dinner frequently at home is associated with consumption of a healthier diet whether or not one is trying to lose weight. Strategies are needed to encourage more cooking among the general population and help infrequent cookers better navigate the food environment outside the home.
\end{abstract}

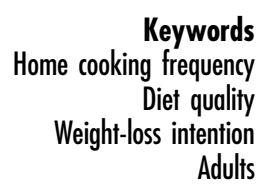

Americans are cooking less and relying more on food prepared away from home, which is typically more energy dense and of lower nutritional value ${ }^{(1-6)}$. Both the decline in cooking and the increase in prepared food consumption are associated with the rise in obesity, which affects approximately one-third of adults ${ }^{(4,6,7)}$. Americans spend less time cooking now than in the past ${ }^{(8-10)}$ and this, in combination with greater reliance on prepared convenience foods, fast foods and other foods prepared away from home, is associated with poorer diet quality ${ }^{(9-11)}$.

A variety of explanations have been identified to explain declines in cooking, including lack of access to healthy foods, time and price constraints and lack of knoweldge or confidence in cooking skills ${ }^{(12,13)}$. Female participation in the workforce outside the home is often cited as a primary determinant of declining cooking frequency as women (and mothers in particular) spend less time cooking than in the past $^{(8,10,14,15)}$. A recent study of demographic patterns in home cooking frequency found that cooking was associated with socio-economic status; adults with low socio-economic status were more likely to cook frequently or not at all ${ }^{(16)}$. To date, little research has focused on the complex relationship between cooking frequency and diet quality an important area of inquiry given evidence suggesting that meals at home increasingly include prepared or semiprepared items ${ }^{(17-19)}$.

Also missing from the literature is research examining whether cooking is associated with weight-loss intention. Several studies have compared the effectiveness of different diets, differences in weight-loss strategies by demographic groups and dieting strategies among individuals trying to lose weight ${ }^{(20-22)}$. In general, this literature has shown that individuals trying to lose weight rely mostly on eating more healthily, reducing energy intake or increasing physical activity. The extent to which cooking is used to achieve these goals is unknown. In the present paper, we attempt to partially fill this gap by exploring the relationship between diet quality and cooking frequency by weight-loss intention. 
It is possible that cooking frequency could be positively or negatively related to weight loss. On the positive side, cooking can provide a high level of control over the ingredients and techniques used in food preparation. On the negative side, not all cooking is healthy and cooking as a weight-loss activity depends on an individual's ability to use healthy ingredients and techniques (e.g. grilling or steaming $v$. deep frying or sautéing). Understanding the relationship between cooking frequency and weight-loss intention is particularly timely given that meal preparation at home is increasingly being promoted as an obesity reduction measure ${ }^{(9,23)}$.

The primary purpose of the study was to examine the association between cooking frequency and diet quality. The secondary aim was to examine patterns in cooking frequency and diet quality by weight-loss intention - which, to our knowledge, has not been previously examined in the literature. We hypothesized that individuals who live in households where dinner is cooked more frequently would consume a healthier diet and that individuals trying to lose weight would cook more frequently than individuals not trying to lose weight.

\section{Methods}

\section{Data and design}

Data were obtained from the consumer behaviour module of the National Health and Nutrition Examination Survey (NHANES), which was added in 2007. The NHANES is a cross-sectional, nationally representative, populationbased survey designed to collect information on the health status, nutritional intake and health-related behaviours of the US population. Participants are selected based on a multistage, clustered, probability sampling strategy ${ }^{(24)}$. Because the NHANES is not solely focused on food and diet, respondents are not necessarily the primary food provider for the household, but during the interview they answer questions about both individual and household behaviours and characteristics.

The present analysis combined data from two waves of data collection (2007-2010) to look at overall patterns during that time period. The 2009-2010 wave of data collection was the most recent data available at the time of analysis. A complete description of data-collection procedures and analytic guidelines are available elsewhere (www.cdc.gov/nchs/nhanes.htm). Analysis was restricted to data from 2007-2010 based on the availability of key variables of interest.

\section{Study sample}

The study sample included adults aged 20 years and older with complete and reliable $24 \mathrm{~h}$ dietary recalls (as determined by the NHANES staff). Survey respondents were excluded if they were pregnant or had diabetes at the time of data collection ( $n$ 1491). The final analytic sample included 9569 adults.

\section{Measures}

Cooking frequency status

Cooking frequency was assessed by the survey question, 'During the past seven days, how many times did you or someone else in your family cook food for dinner or supper at home?' Nine individuals who responded with a frequency greater than 7 and 112 missing values were excluded from analysis. Cooking frequency was categorized into three groups based on cut-off points in the data and the definition in existing literature ${ }^{(16)}$ : low (0-1 times/ week, $n$ 802), medium (2-5 times/week, $n$ 3704) and high (6-7 times/week, $n$ 5063). The mean value for cooking frequency measure was $5 \cdot 1$, the median was $6 \cdot 0$, and less than $5 \%(n 520)$ of respondents did not cook dinner at all. As a quality check, we conducted a series of sensitivity analyses using different iterations of the cooking frequency measure to confirm robustness of this definition. These are described in more detail below.

\section{Energy intake and diet quality}

The dietary recall was conducted during an in-person interview with a trained NHANES health interview professional in a mobile examination centre. During the interview, survey respondents reported detailed descriptions of the type and quantity of all foods and beverages consumed in the $24 \mathrm{~h}$ period prior to the interview, as well as the consumption occasion and location (at home or away from home). NHANES does not collect data regarding whether or not the food was cooked at home or not. Following the interview, NHANES staff used the US Department of Agriculture's Food and Nutrient Database to systematically code the energy and nutrient contents of all foods and beverages consumed.

Diet quality was measured by total kilojoules (from all food and beverages including alcohol) consumed daily, as well as diet composition (grams of protein, fibre, carbohydrates, fat and sugar daily). Total kilojoules as well as the diet composition measures were examined overall as well as by place of consumption (at home and away from home). To get a more complete picture of diet quality, we additionally assessed four characteristics of eating behaviours: (i) number of meals eaten that were not prepared at home; (ii) frequency of fast-food or pizza consumption; (iii) frequency of consuming ready-to-eat meals; and (iv) frequency of consuming frozen meals or frozen pizzas. The exact question wording and response categories can be found in the Appendix. Correlations between these four variables were checked to confirm they were measuring different eating behaviours (Cronbach's $\alpha=0 \cdot 36$ ). For the purpose of the present analysis, lower overall energy intake and lower consumption of carbohydrates, fat and sugar were considered to be healthier or improved diet quality as 
these are key goals included in the 2010 Dietary Guidelines for Americans ${ }^{(25)}$.

\section{Weight-loss intention}

Weight-loss intention was determined by self-reported intentional weight loss of $\geq 4.5 \mathrm{~kg}(\geq 10 \mathrm{lb})$ in the past year or an affirmative response to the question, 'During the past 12 months, have you tried to lose weight?' In the NHANES, respondents are first asked if they had intentional weight loss of $\geq 4.5 \mathrm{~kg}$ ( $\geq 10 \mathrm{lb}$ ); if they respond affirmatively, they are instructed to skip the next question regarding whether or not they were trying to lose weight. By using both questions to define weight-loss intention, we capture individuals who succeeded in losing $\geq 4.5 \mathrm{~kg}$ ( $\geq 10 \mathrm{lb}$ ) as well as those who were trying to lose weight but lost $<4.5 \mathrm{~kg}(<10 \mathrm{lb})$.

\section{Socio-economic and demographic study covariates}

Covariates for the present analysis included body weight, gender, race/ethnicity (non-Hispanic White, non-Hispanic Black, Hispanic, other), age (20-44, 45-64, $\geq 65$ years), education (less than high school, high school or General Educational Development (GED), more than high school), marital status (married, previously married, living with a partner, never married), employment status (not employed (including unemployed, retirees and those not actively looking for work), part time (1-34h/week), full time $(\geq 35 \mathrm{~h} /$ week)), country of birth (US born $v$. born in another country), household size (1-3 persons, $\geq 4$ persons), household food security and income $(\leq 130 \%$ and $>130 \%$ of the federal poverty level). To account for differences in consumption patterns during the week, we also included whether the day of dietary recall was a weekend or weekday. NHANES measures height and body weight during the physical examination component of the survey. These measures are converted into BMI and body weight categories are defined at a BMI of $18.50-24.99 \mathrm{~kg} / \mathrm{m}^{2}$ (healthy), $25.00-29.99 \mathrm{~kg} / \mathrm{m}^{2}$ (overweight) and $\geq 30.00 \mathrm{~kg} / \mathrm{m}^{2}$ (obese) ${ }^{(26)}$. Household food security is measured in NHANES via an eighteen-question questionnaire and then categorized (by the NHANES staff) based on those measures into four categories: full, marginal, low and very low food security ${ }^{(27)}$. For the present analysis, the single low and very low categories were collapsed based on the cut-off points in the data.

\section{Analysis}

All analyses used appropriate survey weights to account for the unequal probability of being selected due to the complex sampling strategy employed by NHANES. Analyses were performed using the statistical software package STATA version 12. Multivariate linear models adjusted for the covariates described above were used to estimate the relationship between cooking frequency (defined as low (0-1 times/week), medium (2-5 times/ week) and high (6-7 times/week)) and diet quality - the primary aim of the paper. To address our secondary aim, we estimated models including an interaction term between weight-loss intention and cooking frequency to identify whether individuals trying to lose weight cooked at a different frequency from those who are not. All covariates were included based on prior literature suggesting a relationship with the outcome variables and cooking frequency, regardless of significance ${ }^{(12,16)}$. We used multinomial logistic regression to estimate the relative risk ratio (RRR) of being in the low or medium cooking category as compared with the high cooking category adjusting for demographic characteristics. To test the robustness of the cooking measure, we replicated the analyses above using three definitions of cooking frequency: (i) an ordinal, count variable ranging from 0 to 7 dinners/week; (ii) a dichotomous variable with low defined as 0-2 times/week and high as 3-7 times/week; and (iii) a three-category variable with the categories of low (0-2 times/week), medium (3-5 times/week) and high (6-7 times/week). Significance of all differences between groups was considered at $P<0 \cdot 05$, and all tests were two-sided. Tables 3 and 4 report predicted means based on the adjusted models.

\section{Results}

Table 1 presents the characteristics of the study sample overall and by household cooking frequency. Overall, among American adults 20 years or older in 2007-2010, $8 \%$ lived in households where someone cooked dinner 0-1 times/week, $44 \%$ lived in households where someone cooked dinner 2-5 times/week and $48 \%$ lived in households where someone cooked dinner 6-7 times/week. Differences between the cooking categories by race/ethnicity, age, education, marital status, employment status, country of birth, household size, food security and income level were significant at $P<0.001$.

In addition to these bivariate relationships we also estimated the adjusted relationship between demographic characteristics and household cooking frequency to understand the relative risk of being in the low or medium cooking category as compared with the high category (Table 2). We found that compared with Whites, Blacks were more likely to live in households where dinner is cooked with low $(\mathrm{RRR}=2.36, \quad P<0.001)$ or medium (RRR $=1.55, P<0.001)$ frequency, and Hispanics were less likely to live in households where dinner is cooked with medium frequency $(\mathrm{RRR}=0.72, P<0.01)$ than with high frequency. Compared with females, males were slightly more likely to be in the low frequency category $(\mathrm{RRR}=1 \cdot 26$, $P<0.05)$ and slightly less likely to be in the medium frequency category $(\mathrm{RRR}=0 \cdot 89, P<0 \cdot 05)$. Being previously married $(\mathrm{RRR}=3 \cdot 15, P<0 \cdot 001)$ or never married $(\mathrm{RRR}=$ $3.25, P<0.001)$ and working $\geq 35 \mathrm{~h} /$ week $(\mathrm{RRR}=1.53$, $P<0.001)$ were associated with greater risk of being in the low cooking frequency category. Having high income was 
Table 1 Characteristics of the study population according to cooking frequency: US adults (aged $\geq 20$ years) in the National Health and Nutrition Examination Survey (NHANES), 2007-2010†

\begin{tabular}{|c|c|c|c|c|c|c|c|c|c|}
\hline & \multicolumn{2}{|c|}{ Total } & \multicolumn{2}{|c|}{$\begin{array}{c}\text { Low cooking } \\
(0-1 \text { times/week }) \ddagger\end{array}$} & \multicolumn{2}{|c|}{$\begin{array}{l}\text { Medium cooking } \\
(2-5 \text { times/week }) \ddagger\end{array}$} & \multicolumn{2}{|c|}{$\begin{array}{c}\text { High cooking } \\
\text { (6-7 times/week)‡ }\end{array}$} & \multirow[b]{2}{*}{$P$ for difference } \\
\hline & $n$ & $\%$ & $n$ & $\%$ & $n$ & $\%$ & $n$ & $\%$ & \\
\hline Total & 9569 & 100 & 802 & 8 & 3704 & 44 & 5063 & 48 & \\
\hline \multicolumn{10}{|l|}{ Dieting status } \\
\hline Trying to lose weight & 3839 & 43 & 337 & 42 & 1617 & 46 & 1885 & 40 & \multirow[t]{2}{*}{0.003} \\
\hline Not trying to lose weight & 5726 & 57 & 468 & 58 & 2087 & 54 & 3174 & 60 & \\
\hline \multicolumn{10}{|l|}{ Body weight§ } \\
\hline Healthy & 2719 & 32 & 219 & 30 & 1025 & 31 & 1475 & 33 & \multirow[t]{3}{*}{0.181} \\
\hline Overweight & 3334 & 35 & 273 & 33 & 1306 & 36 & 1755 & 35 & \\
\hline Obese & 3269 & 33 & 289 & 37 & 1289 & 33 & 1691 & 32 & \\
\hline \multicolumn{10}{|l|}{ Sex } \\
\hline Male & 4710 & 49 & 429 & 53 & 1770 & 48 & 2511 & 49 & \multirow[t]{2}{*}{0.051} \\
\hline Female & 4859 & 51 & 373 & 47 & 1934 & 52 & 2552 & 51 & \\
\hline \multicolumn{10}{|l|}{ Race/ethnicity } \\
\hline Non-Hispanic White & 4739 & 75 & 337 & 66 & 1985 & 78 & 2417 & 73 & \multirow[t]{4}{*}{$<0.001$} \\
\hline Non-Hispanic Black & 1742 & 11 & 229 & 19 & 870 & 13 & 643 & 9 & \\
\hline Hispanic & 2651 & 14 & 207 & 15 & 712 & 9 & 1732 & 19 & \\
\hline Other & 437 & 6 & 29 & 5 & 137 & 5 & 271 & 8 & \\
\hline \multicolumn{10}{|l|}{ Age } \\
\hline 20-44 years & 4309 & 49 & 365 & 50 & 1843 & 53 & 2101 & 45 & \multirow[t]{3}{*}{$<0.001$} \\
\hline $45-64$ years & 3146 & 36 & 245 & 33 & 1220 & 35 & 1681 & 36 & \\
\hline$\geq 65$ years & 2114 & 15 & 192 & 17 & 641 & 11 & 1281 & 18 & \\
\hline \multicolumn{10}{|l|}{ Education } \\
\hline Less than high school & 2651 & 18 & 202 & 20 & 694 & 12 & 1755 & 23 & \multirow[t]{3}{*}{$<0.001$} \\
\hline High school (or GED) & 2292 & 24 & 201 & 25 & 926 & 24 & 1165 & 24 & \\
\hline More than high school & 4614 & 58 & 397 & 55 & 2082 & 64 & 2135 & 52 & \\
\hline \multicolumn{10}{|l|}{ Marital status } \\
\hline Currently married & 4976 & 56 & 246 & 33 & 1848 & 55 & 2882 & 61 & \multirow[t]{4}{*}{$<0.001$} \\
\hline Previously married & 2101 & 17 & 275 & 29 & 793 & 17 & 1033 & 16 & \\
\hline Living with a partner & 765 & 8 & 44 & 5 & 317 & 8 & 404 & 8 & \\
\hline Never married & 1724 & 19 & 237 & 32 & 743 & 20 & 744 & 15 & \\
\hline \multicolumn{10}{|l|}{ Employment status } \\
\hline Not employed & 4065 & 35 & 336 & 35 & 1289 & 28 & 2440 & 41 & $<0.001$ \\
\hline Part time (1-34 h/week) & 1266 & 14 & 103 & 13 & 524 & 15 & 639 & 14 & \\
\hline Full time ( $\geq 35 \mathrm{~h} /$ week $)$ & 4017 & 51 & 337 & 52 & 1774 & 57 & 1906 & 45 & \\
\hline Country of birth & & & & & & & & & \\
\hline Born in the USA & 7111 & 83 & 629 & 85 & 3116 & 89 & 3366 & 77 & $<0.001$ \\
\hline Born in another country & 2457 & 17 & 173 & 15 & 588 & 11 & 1696 & 23 & \\
\hline Household size & & & & & & & & & \\
\hline $1-3$ persons & 5864 & 64 & 606 & 78 & 2425 & 68 & 2833 & 59 & $<0.001$ \\
\hline$\geq 4$ persons & 3705 & 36 & 196 & 22 & 1279 & 32 & 2230 & 41 & \\
\hline Food security & & & & & & & & & \\
\hline Full food security & 6801 & 79 & 603 & 80 & 2764 & 82 & 3434 & 77 & 0.001 \\
\hline Marginal food security & 1002 & 8 & 70 & 7 & 334 & 6 & 598 & 10 & \\
\hline Low/very low food security & 1758 & 13 & 128 & 12 & 602 & 11 & 1028 & 14 & \\
\hline Income level & & & & & & & & & \\
\hline Low income $\leq 130 \% \mathrm{FPL}$ & 2768 & 19 & 233 & 22 & 818 & 14 & 1717 & 24 & $<0.001$ \\
\hline High income $>130 \% \mathrm{FPL}$ & 6801 & 81 & 569 & 78 & $2 \cdot 886$ & 86 & 3346 & 76 & \\
\hline
\end{tabular}

GED, General Educational Development; FPL, federal poverty level.

$P$ value for difference is based on the $X^{2}$ test.

†Percentage of the US population estimated with survey weights to adjust for unequal probability of sampling

$\ddagger$ Cooking frequency is the number of times per week the respondent or someone in the household cooked dinner.

$\S$ Healthy weight, $\mathrm{BMI}=18.50-24.99 \mathrm{~kg} / \mathrm{m}^{2}$; overweight, $\mathrm{BMI}=25.00-29.99 \mathrm{~kg} / \mathrm{m}^{2}$; obese, $\mathrm{BMI} \geq 30.00 \mathrm{~kg} / \mathrm{m}^{2}$.

associated with reduced risk of being in the medium category $(\mathrm{RRR}=0.60, P<0.001)$ compared with having low income. Compared with being unemployed, working part time $(\mathrm{RRR}=1.39, \quad P<0.001)$ and full time $(\mathrm{RRR}=1.55$, $P<0.001)$ were associated with increased risk of being in the medium cooking category. Compared with having less than high-school education, a high-school diploma $(\mathrm{RRR}=1.39$, $P<0.01)$ and greater than high-school education (RRR $=$ $1 \cdot 60, P<0.001)$ were associated with greater risk of being in the medium cooking category.
Table 3 presents the predicted per capita kilojoules and diet quality measures by household cooking frequency. People in the high cooking category consumed significantly fewer total kilojoules on the surveyed day than those in the low category (9054v. $9627 \mathrm{~kJ} / \mathrm{d}, P=0.002)$. The difference between kilojoules consumed at home for people living in high $v$. low cooking households (6163 $v$. $5406 \mathrm{~kJ} / \mathrm{d}, \quad P=0.001)$ was smaller than the difference between the kilojoules consumed away from home (2891 v. $4217 \mathrm{~kJ} / \mathrm{d}, P<0.001)$. Compared with those in the low 
Table 2 Predictors of cooking frequency† among the study population: US adults (aged $\geq 20$ years) in the National Health and Nutrition Examination Survey (NHANES), 2007-2010

\begin{tabular}{|c|c|c|c|c|}
\hline & \multicolumn{2}{|c|}{$\begin{array}{l}\text { Low cooking (0-1 times/week) } \\
\text { (Ref: high cooking (6-7 times/week)) }\end{array}$} & \multicolumn{2}{|c|}{$\begin{array}{l}\text { Medium cooking (2-5 times/week) } \\
\text { (Ref: high cooking (6-7 times/week) }\end{array}$} \\
\hline & RRR & $95 \% \mathrm{Cl}$ & RRR & $95 \% \mathrm{Cl}$ \\
\hline \multicolumn{5}{|l|}{ Dieting status (Ref: not trying) } \\
\hline Trying to lose weight & 1.07 & $0.85,1.34$ & 1.08 & $0.94,1.24$ \\
\hline \multicolumn{5}{|l|}{ Body weight (Ref: healthy) } \\
\hline Overweight & 1.04 & $0.82,1.31$ & $1 \cdot 16$ & $0.99,1.36$ \\
\hline Obese & 1.24 & $0.94,1.64$ & $1 \cdot 14$ & $0.96,1.35$ \\
\hline \multicolumn{5}{|l|}{ Sex (Ref: female) } \\
\hline Male & $1 \cdot 26^{*}$ & $1.06,1.53$ & $0.89^{*}$ & $0.81,0.98$ \\
\hline \multicolumn{5}{|l|}{ Race/ethnicity (Ref: White) } \\
\hline Non-Hispanic Black & $2 \cdot 36^{\star \star \star}$ & $1 \cdot 74,3 \cdot 19$ & $1.55^{\star \star \star}$ & $1.33,1.81$ \\
\hline Hispanic & 1.38 & $0.94,2.02$ & $0.72^{\star *}$ & $0.58,0.89$ \\
\hline Other & 0.83 & $0.43,1.61$ & 0.78 & $0.56,1.09$ \\
\hline \multicolumn{5}{|l|}{ Age (Ref: $20-44$ years) } \\
\hline $45-64$ years & 0.74 & $0.54,1.02$ & $0.68^{\star * \star}$ & $0.59,0.78$ \\
\hline$\geq 65$ years & $0 \cdot 81$ & $0.54,1.20$ & $0.50^{\star \star \star}$ & $0.41,0.61$ \\
\hline \multicolumn{5}{|c|}{ Education (Ref: less than high school) } \\
\hline High school (or GED) & 1.09 & $0.83,1.43$ & $1 \cdot 39^{\star \star}$ & $1 \cdot 13,1.70$ \\
\hline More than high school & 1.08 & $0.79,1.46$ & $1.60^{\star \star \star}$ & $1.32,1.93$ \\
\hline \multicolumn{5}{|l|}{ Marital status (Ref: married) } \\
\hline Previously married & $3 \cdot 15^{\star \star \star}$ & $2 \cdot 42,4 \cdot 11$ & $1 \cdot 38^{\star *}$ & $1.13,1.69$ \\
\hline Living with a partner & 1.05 & $0.58,1.88$ & 1.25 & $0.96,1.61$ \\
\hline Never married & $3 \cdot 25^{\star \star \star}$ & $2.26,4.69$ & $1 \cdot 28^{*}$ & $1.06,1.55$ \\
\hline \multicolumn{5}{|c|}{ Employment (Ref: not employed) } \\
\hline Part time $(1-34 \mathrm{~h} /$ week) & 1.24 & $0.85,1.80$ & $1 \cdot 39^{\star \star \star}$ & $1.15,1.68$ \\
\hline Full time $(>35 \mathrm{~h} /$ week $)$ & $1.53^{\star * *}$ & $1.22,1.93$ & $1.55^{\star * *}$ & $1.34,1.79$ \\
\hline \multicolumn{5}{|c|}{ Country of birth (Ref: foreign born) } \\
\hline Born in the USA & 1.38 & $0.94,2.02$ & $1 \cdot 85^{\star \star \star}$ & $1.41,2.42$ \\
\hline \multicolumn{5}{|c|}{ Household size (Ref: $1-3$ persons) } \\
\hline$\geq 4$ persons & $0.41^{\star \star \star}$ & $0.29,0.58$ & $0.66^{\star \star}$ & $0.52,0.85$ \\
\hline \multicolumn{5}{|l|}{ Food security (Ref: full security) } \\
\hline Marginal food security & 0.63 & $0.38,1.04$ & $0.69^{* *}$ & $0.53,0.88$ \\
\hline Low/very low food security & 0.75 & $0.53,1.05$ & $1 \cdot 10$ & $0.83,1.47$ \\
\hline \multicolumn{5}{|c|}{ Income status (ref: Low, $\leq 130 \% \mathrm{FPL}$ ) } \\
\hline High $(>130 \%$ FPL $)$ & 0.86 & $0.71,1.05$ & $0.60^{\star \star \star}$ & $0.51,0.71$ \\
\hline
\end{tabular}

Ref, reference category; RRR relative risk ratio; GED, General Educational Development; FPL, federal poverty level.

Multinomial logistic regression was used to adjust for age, sex, race/ethnicity, education, body weight category, weight-loss intention, marital status, employment status, if born in the USA, household size, household food security and income level.

${ }^{\star} P<0.10,{ }^{* \star} P<0.05,{ }^{* \star \star} P<0.01$.

†Cooking frequency is the number of times per week the respondent or someone in the household cooked dinner.

cooking category, those in the high cooking category consumed significantly more grams of fibre $(16.9 v .15 .9 \mathrm{~g} / \mathrm{d}$, $P=0.033)$ and fewer grams of carbohydrates $(262 v .284 \mathrm{~g} / \mathrm{d}$, $P<0.001)$, fat (81 v. $86 \mathrm{~g} / \mathrm{d}, P=0.016)$ and sugar (119 v. $135 \mathrm{~g} / \mathrm{d}, P<0 \cdot 001)$. Compared with the low cooking frequency category, individuals in the high category consumed significantly fewer meals not from home per week $(2 \cdot 8 v \cdot 6 \cdot 7$ meals/week, $P<0 \cdot 001)$, fast-food meals per week (1.6v. 3.4 meals/week, $P<0.001)$, frozen meals/pizzas in the past $30 \mathrm{~d}$ (2.4 v. 3.6 meals, $P<0.001)$ and meals comprised of readyto-eat foods in the past $30 \mathrm{~d}(1.4 v .2 .5$ meals, $P<0.001)$.

Individuals living in households with medium cooking frequency consumed fewer total kilojoules (9222 $v$. $9627 \mathrm{~kJ} / \mathrm{d}, P=0.05)$ and kilojoules away from home $(3573$ v. $4217 \mathrm{~kJ} / \mathrm{d}, P=0.019)$ compared with individuals in the low cooking category. Compared with people in the low cooking category, those in the medium category consumed fewer carbohydrates $(265 v .284 \mathrm{~g} / \mathrm{d}, P=0 \cdot 012)$ and less sugar (121 v. $135 \mathrm{~g} / \mathrm{d}, P=0 \cdot 006)$. Medium cookers also consumed fewer meals not from home per week
( $4.5 v .6 .7$ meals/week, $P<0.001$ ), less fast food per week $(2 \cdot 1 v .3 .4$ meals/week, $P<0 \cdot 001)$ and fewer frozen meals/ pizzas in the past $30 \mathrm{~d}(3.0 v .3 .6$ meals, $P=0.035)$ compared with low cookers.

Table 4 presents the association between household cooking frequency and diet quality by weight-loss intention. Overall, people trying to lose weight consumed a healthier diet than people not trying to lose weight, regardless of how frequently they, or someone in their household, cooked. Higher cooking frequency was associated with improved diet quality among individuals both trying and not trying to lose weight. Within each cooking category, individuals trying to lose weight had better diet quality than those not trying to lose weight. Among low cookers, individuals trying to lose weight consumed significantly fewer kilojoules at home (4862v. $5832 \mathrm{~kJ} / \mathrm{d}, P<0.023$ ), grams of carbohydrates (264 v. $299 \mathrm{~g} / \mathrm{d}, P=0.020)$ and grams of sugar $(125 \mathrm{~g} v$. $143 \mathrm{~g} / \mathrm{d}, P=0.032)$ than individuals who were not trying to lose weight. Among individuals with medium household cooking frequency, those trying to lose weight consumed 
Table 3 Predicted per capita energy consumption $(\mathrm{kJ}) \dagger$ and diet quality measures according to cooking frequency: US adults (aged $\geq 20$ years) in the National Health and Nutrition Examination Survey (NHANES), 2007-2010

\begin{tabular}{|c|c|c|c|c|c|c|c|c|}
\hline & \multicolumn{2}{|c|}{ All } & \multicolumn{2}{|c|}{$\begin{array}{c}\text { Low cooking } \\
(0-1 \text { times/week }) \ddagger\end{array}$} & \multicolumn{2}{|c|}{$\begin{array}{l}\text { Medium cooking } \\
(2-5 \text { times/week) } \ddagger\end{array}$} & \multicolumn{2}{|c|}{$\begin{array}{c}\text { High cooking } \\
(6-7 \text { times/week }) \ddagger\end{array}$} \\
\hline & Mean & $\mathrm{SE} \S$ & Mean & $\mathrm{SE}$ & Mean & SE & Mean & SE \\
\hline Mean total energy consumption (total kJ/d) & 9171 & 67 & $9627 \|, \emptyset$ & 180 & 9222 & 88 & 9054 & 113 \\
\hline Consumed at home $(\mathrm{kJ} / \mathrm{d})$ & 5879 & 67 & $5406 \|$ & 213 & $5648 \|$ & 92 & 6163 & 92 \\
\hline Consumed away from home (kJ/d) & 3293 & 50 & $4217 \|, \rrbracket$ & 226 & $3573 \|$ & 96 & 2891 & 71 \\
\hline Mean total grams of protein $(\mathrm{g} / \mathrm{d})$ & 84.0 & 0.7 & 84.0 & 1.9 & 84.0 & 0.8 & $84 \cdot 0$ & $1 \cdot 1$ \\
\hline Mean total grams of fibre $(\mathrm{g} / \mathrm{d})$ & $16 \cdot 5$ & 0.2 & $15 \cdot 9 \|$ & 0.5 & $16 \cdot 2 \|$ & 0.3 & $16 \cdot 9$ & 0.4 \\
\hline Mean total grams of carbohydrates $(\mathrm{g} / \mathrm{d})$ & 265 & $2 \cdot 0$ & 284\|, & $6 \cdot 0$ & 265 & 3.0 & 262 & 3.0 \\
\hline Mean total grams of fat $(\mathrm{g} / \mathrm{d})$ & 82 & 0.8 & $86 \|$ & 1.9 & 83 & 1.0 & 81.0 & 1.3 \\
\hline Mean total grams of sugar $(\mathrm{g} / \mathrm{d})$ & 121 & 1.4 & $135 \|, \emptyset$ & $4 \cdot 2$ & 121 & $2 \cdot 2$ & 119 & 2.2 \\
\hline Mean no. of meals not from home per week & 3.8 & 0.0 & $6 \cdot 7 \|, 9$ & 0.3 & $4.5 \|$ & 0.1 & $2 \cdot 8$ & 0.1 \\
\hline Mean no. of meals from fast food per week & $2 \cdot 0$ & 0.0 & $3 \cdot 4 \|, \Phi$ & 0.2 & $2 \cdot 1 \|$ & 0.1 & 1.6 & 0.1 \\
\hline Mean no. of frozen meals/pizzas in past $30 \mathrm{~d}$ & $2 \cdot 7$ & 0.1 & $3.6 \|, \mathbb{9}$ & 0.2 & $3.0 \|$ & 0.2 & $2 \cdot 4$ & 0.1 \\
\hline Mean no. of ready-to-eat meals in past $30 \mathrm{~d}$ & 1.8 & 0.1 & $2.5 \|$ & 0.3 & $2 \cdot 2 \|$ & 0.1 & 1.4 & 0.1 \\
\hline
\end{tabular}

Multivariate regression was used to adjust for age, sex, race/ethnicity, education, body weight category, weight-loss intention, marital status, employment status, if born in the USA, household size, household food security income level and day of the week.

†Kilojoules results converted from kilocalories; $1 \mathrm{kcal}=4 \cdot 184 \mathrm{~kJ}$.

$\ddagger$ Cooking frequency is the number of times per week the respondent or someone in the household cooked dinner.

$\S$ Standard errors $<0.05$ rounded to 0.0 .

IIMean value was significantly different from that of high cookers $(P<0.05)$.

IMean value was significantly different from that of medium cookers $(P<0.05)$.

Table 4 Predicted per capita energy consumption $(\mathrm{kJ}) \dagger$ and diet quality measures by cooking frequency and weight-loss intention: US adults (aged $\geq 20$ years) in the National Health and Nutrition Examination Survey (NHANES), 2007-2010

\begin{tabular}{|c|c|c|c|c|c|c|c|c|c|c|c|c|c|c|c|c|}
\hline & \multicolumn{4}{|c|}{ All } & \multicolumn{4}{|c|}{$\begin{array}{c}\text { Low cooking } \\
(0-1 \text { times/week }) \ddagger\end{array}$} & \multicolumn{4}{|c|}{$\begin{array}{l}\text { Medium cooking } \\
(2-5 \text { times/week }) \ddagger\end{array}$} & \multicolumn{4}{|c|}{$\begin{array}{c}\text { High cooking } \\
(6-7 \text { times/week }) \ddagger\end{array}$} \\
\hline & \multicolumn{2}{|c|}{$\begin{array}{l}\text { Tried } \\
\text { to lose } \\
\text { weight }\end{array}$} & \multicolumn{2}{|c|}{$\begin{array}{l}\text { Did not try } \\
\text { to lose } \\
\text { weight }\end{array}$} & \multicolumn{2}{|c|}{$\begin{array}{c}\text { Tried to } \\
\text { lose } \\
\text { weight }\end{array}$} & \multicolumn{2}{|c|}{$\begin{array}{l}\text { Did not try } \\
\text { to lose } \\
\text { weight }\end{array}$} & \multicolumn{2}{|c|}{$\begin{array}{c}\text { Tried to } \\
\text { lose } \\
\text { weight }\end{array}$} & \multicolumn{2}{|c|}{$\begin{array}{l}\text { Did not try } \\
\text { to lose } \\
\text { weight }\end{array}$} & \multicolumn{2}{|c|}{$\begin{array}{l}\text { Tried } \\
\text { to lose } \\
\text { weight }\end{array}$} & \multicolumn{2}{|c|}{$\begin{array}{l}\text { Did not try } \\
\text { to lose } \\
\text { weight }\end{array}$} \\
\hline & Mean & SE & Mean & SE & Mean & SE & Mean & SE & Mean & SE & Mean & SE & Mean & $\mathrm{SE}$ & Mean & SE \\
\hline $\begin{array}{l}\text { Mean total energy consumption } \\
(\text { total } \mathrm{kJ} / \mathrm{d})\end{array}$ & $8832 \S$ & 88 & 9544 & 92 & $9180 \|$ & 264 & 9975 & 293 & $8807 \S$ & 134 & 9548 & 105 & $8632 \S$ & 113 & 9381 & 138 \\
\hline Consumed at home $(\mathrm{kJ} / \mathrm{d})$ & $5489 \S$ & 105 & 6247 & 96 & $4862 \S, \|$ & 297 & $5832 \|$ & 293 & $5234 \S, \|$ & 113 & $5966 \|$ & 130 & $5724 \S$ & 117 & 6502 & 117 \\
\hline $\begin{array}{l}\text { Consumed away from home } \\
(\mathrm{kJ} / \mathrm{d})\end{array}$ & 3343 & 117 & 3297 & 71 & $4318 \|$ & 360 & $4142 \|$ & 318 & $3569 \|$ & 130 & $3577 \|$ & 117 & 2908 & 117 & 2879 & 67 \\
\hline $\begin{array}{l}\text { Mean total grams of protein } \\
(\mathrm{g} / \mathrm{d})\end{array}$ & $81 \S$ & 1.0 & 86 & 1.0 & 82 & $2 \cdot 6$ & 86 & 3.0 & $81 \S$ & $1 \cdot 2$ & 86 & $1 \cdot 4$ & $81 \S$ & $1 \cdot 4$ & 86 & 1.3 \\
\hline Mean total grams of fibre $(\mathrm{g} / \mathrm{d})$ & 16 & 0.3 & 16 & 0.3 & $15 \|$ & 0.5 & 16 & 0.6 & 16 & 0.3 & $16 \|$ & 0.3 & 17 & 0.4 & 17 & 0.4 \\
\hline $\begin{array}{l}\text { Mean total grams of } \\
\text { carbohydrates }(\mathrm{g} / \mathrm{d})\end{array}$ & $252 \S$ & 2 & 277 & 3 & $265 \S, \|$ & 8 & $299 \|, \mathbb{1}$ & 10 & $251 \S$ & 4 & 276 & 3 & $248 \S$ & 4 & 273 & 4 \\
\hline Mean total grams of fat $(\mathrm{g} / \mathrm{d})$ & $80 \S$ & 1 & 86 & 1 & 82 & 3 & 89 & 3 & $79 \S$ & 1 & 86 & 1 & $77 \S$ & 1 & 83 & 2 \\
\hline Mean total grams of sugar $(\mathrm{g} / \mathrm{d})$ & $114 \S$ & 2 & 130 & 2 & $125 \S, \|$ & 6 & $143 \|, \rrbracket$ & 6 & $112 \S$ & 3 & 127 & 3 & $111 \S$ & 3 & 126 & 3 \\
\hline $\begin{array}{l}\text { Mean no. of meals not from home } \\
\text { per week }\end{array}$ & 3.9 & 0.1 & $3 \cdot 8$ & 0.1 & $6 \cdot 8 \|, \pi$ & 0.4 & $6 \cdot 5 \|, \mathbb{\Upsilon}$ & 0.3 & $4 \cdot 6 \|$ & $0 \cdot 1$ & $4 \cdot 4 \|$ & 0.1 & $2 \cdot 7^{\circ}$ & 0.1 & $2 \cdot 8$ & 0.7 \\
\hline $\begin{array}{l}\text { Mean no. of meals from fast food } \\
\text { per week }\end{array}$ & $1.9 \S$ & 0.1 & $2 \cdot 1$ & $0 \cdot 1$ & $3 \cdot 2 \|, \mathbb{1}$ & 0.3 & $3.6 \|, \mathbb{9}$ & 0.3 & $2 \cdot 1 \|$ & 0.1 & $2 \cdot 1 \|$ & 0.1 & $1.5 \S$ & 0.1 & $1 \cdot 8$ & 0.1 \\
\hline $\begin{array}{l}\text { Mean no. of frozen meals/pizzas } \\
\text { in past } 30 \mathrm{~d}\end{array}$ & $3.0 \S$ & 0.2 & $2 \cdot 6$ & 0.1 & $4.0 \|$ & 0.4 & $3 \cdot 3 \|$ & 0.3 & $3 \cdot 4 \S, \|$ & 0.3 & $2 \cdot 6$ & 0.2 & $2 \cdot 3$ & 0.1 & $2 \cdot 4$ & 0.2 \\
\hline $\begin{array}{l}\text { Mean no. of ready-to-eat meals in } \\
\text { past } 30 \mathrm{~d}\end{array}$ & 1.9 & 0.1 & $1 \cdot 8$ & 0.1 & $2 \cdot 4 \|$ & 0.5 & $2 \cdot 5 \|$ & 0.3 & $2 \cdot 3 \|$ & 0.2 & $2 \cdot 0 \|$ & 0.1 & 1.3 & 0.1 & 1.4 & 0.1 \\
\hline
\end{tabular}

Multivariate regression was used to adjust for age, sex, race/ethnicity, education, body weight category, weight-loss intention, marital status, employment status, if born in the USA, income level, household size, household food security and day of the week.

tKilojoules results converted from kilocalories; $1 \mathrm{kcal}=4.184 \mathrm{~kJ}$.

¥Cooking frequency is the number of times per week the respondent or someone in the household cooked dinner.

$\S$ Mean value was significantly different from that of persons in the same cooking category who did not try to lose weight $(P<0.05)$.

IIMean value was significantly different from that of high cookers in the same weight-loss intention category $(P<0.05)$.

đMean value was significantly different from that of medium cookers in the same weight-loss intention category $(P<0.05)$.

fewer total kilojoules (8807 v. $9548 \mathrm{~kJ} / \mathrm{d}, P<0 \cdot 001)$, kilojoules at home $(5234 v .5966 \mathrm{~kJ} / \mathrm{d}, P<0 \cdot 001)$, carbohydrates (251 v. $276 \mathrm{~g} / \mathrm{d}, P<0.001)$, fat $(79 v .86 \mathrm{~g} / \mathrm{d}, P=0.001)$ and sugar $(112 v .127 \mathrm{~g} / \mathrm{d}, P<0 \cdot 001)$ compared with those with medium household cooking frequency who were not trying to lose weight. Medium cookers trying to lose weight 
consumed more frozen meals/pizzas in the past $30 \mathrm{~d}(3.4 v$. 2.6 meals, $P=0.007)$ compared with medium cookers not trying to lose weight. Among those with high household cooking frequency, individuals trying to lose weight consumed fewer total kilojoules (8632 v. $9381 \mathrm{~kJ} / \mathrm{d}, P<0.001)$, kilojoules at home $(5724 v .6502 \mathrm{~kJ} / \mathrm{d}, P<0.001)$, carbohydrates $(248 v .273 \mathrm{~g} / \mathrm{d}, P<0.001)$, fat $(77 v .83 \mathrm{~g} / \mathrm{d}, P<0.001)$, sugar (111 v. $126 \mathrm{~g} / \mathrm{d}, P<0.001)$ and meals from fast food per week (1.5 v. 1.8 meals/week, $P=0.001)$ compared with individuals not trying to lose weight.

Among individuals who were not trying to lose weight, higher household cooking frequency was associated with better diet quality. Compared with the low cooking category, those with high household cooking frequency consumed significantly more kilojoules at home $(6502 v .5832 \mathrm{~kJ} / \mathrm{d}$, $P=0.027)$ and fewer kilojoules away from home (2879 $v$. $4142 \mathrm{~kJ} / \mathrm{d}, P<0 \cdot 001)$, less carbohydrates $(273 v .299 \mathrm{~g} / \mathrm{d}$, $P=0.013)$ and less sugar (126 v.143 g/d, $P=0.008)$. People with high household cooking frequency who not trying to lose weight also consumed fewer meals not from home (2.8 v. 6.5 meals/week, $P<0.001)$, meals from fast food $(1.8 v .3 .6$ meals/week, $P<0.001$ ), frozen meals/pizza in the past $30 \mathrm{~d}$ ( 2.4 v. 3.3 meals, $P=0.014$ ) and ready-to-eat meals in the past $30 \mathrm{~d}(1.4 v .2 .5$ meals, $P=0.004)$ compared with those with low household cooking frequency who were not trying to lose weight. The comparison of low with medium cooking frequency among people not trying to lose weight yielded similar differences. Medium cookers not trying to lose weight consumed fewer grams of carbohydrates $(276 v .299 \mathrm{~g} / \mathrm{d}$, $P=0.047)$, sugar (127v.143 g/d, $P=0.024)$, meals not from home per week ( $4.4 v .6 .5$ meals/week, $P<0.001)$ and meals from fast food $(2.1 v .3 .6$ meals/week, $P<0.001)$ compared with low cookers not trying to lose weight.

Among individuals trying to lose weight, individuals in households that cooked with high frequency consumed more kilojoules at home $(5724 v .4862 \mathrm{~kJ} / \mathrm{d}, P=0.001)$, but fewer total kilojoules $(8632 v .9180 \mathrm{~kJ} / \mathrm{d}, P=0.038)$, kilojoules away from home (2908 v. $4318 \mathrm{~kJ} / \mathrm{d}, P<0.001)$, carbohydrates $(248 v .265 \mathrm{~g} / \mathrm{d}, P=0.037)$ and grams of sugar $(111 v .125 \mathrm{~g} / \mathrm{d}, P=0.021)$ than those trying to lose weight in households with low cooking frequency. People in the high cooking category who were not trying to lose weight also consumed fewer meals not from home per week ( $2.7 v .6 .8$ meals/week, $P<0.001)$, fast-food meals per week (1.5 v. 3.2 meals/week, $P<0.001)$, frozen meals/ pizzas in the past $30 \mathrm{~d}(2.3 v .4 .0$ meals, $P<0.001)$ and fewer ready-to-eat meals in the past $30 \mathrm{~d}(1.3 v .2 .4$ meals, $P=0.045)$ than those in the low cooking category who were trying to lose weight. People in the medium cooking category who were trying to lose weight consumed fewer meals not from home per week ( $4.6 v .6 .8$ meals/week, $P<0 \cdot 001)$ and fewer fast-food meals per week $(2 \cdot 1 v \cdot 3 \cdot 2$ meals/week, $P=0.001)$ than those in the low cooking category who were trying to lose weight.

We also examined the diet composition measures stratified by consumption location (at home or away from home).
This analysis (not shown, but available upon request) showed that the trend for each diet composition measure was similar to the trend for overall energy intake. Specifically, higher cooking frequency was associated with a greater proportion of carbohydrates, fat, fibre, protein and sugar being consumed at home. In separate analyses (see online supplementary material, Supplemental Tables 1 and 2), we explored the relationship between cooking frequency, body weight status (normal, overweight and obese) and diet quality. We did not observe notable significant differences by body weight overall or within or between cooking frequency categories. The robustness checks for our measure of cooking frequency yielded results that were substantively similar to the results presented above. These analyses are available upon request.

\section{Discussion}

The present study examined the relationship between cooking frequency and diet quality and whether that relationship varies by weight loss intention. We estimated this relationship adjusting for covariates related to both cooking behaviour and diet quality. We found that cooking dinner at home more frequently ( $\geq 2$ times/week) was associated with better diet quality, specifically lower consumption of total kilojoules, carbohydrates, fat and sugar, fewer kilojoules away from home, and less consumption of fast food, meals consumed not prepared at home, frozen and ready-to-eat meals. As expected, cooking more frequently is protective regardless of weight-loss intention. In other words, if a person or someone in their household cooks dinner frequently, regardless of whether or not they are trying to lose weight, diet quality improves. This is likely due to the relatively lower energy, fat and sugar contents in foods cooked at home compared with convenience foods or foods consumed away from home ${ }^{(28)}$. Inconsistent with our hypotheses was the finding that individuals trying to lose weight did not cook more frequently than those not trying to lose weight. These study results are consistent with prior research indicating that substituting meals from away-from-home sources with home cooked meals is associated with better diet quality ${ }^{(2,29)}$. Unlike previous research suggesting that healthy-weight people spend more time cooking than overweight or obese people, we found no significant relationship between cooking frequency and body weight status ${ }^{(30)}$.

Our finding that people living in households with higher cooking frequency generally follow a healthier diet than people living in households with low cooking frequency, regardless of whether they are trying to lose weight, has important implications for obesity prevention efforts. In particular, it is important to consider how to support and incentivize greater cooking frequency within the constraints of modern life. This might include teaching cooking skills from an early age by re-introducing a home 
economics curriculum in schools to build cooking skills, knowledge and confidence ${ }^{(31-33)}$. Inclusion of cooking skills into nutrition education has been shown to be effective at improving cooking self-efficacy, fostering healthy eating habits, and is an important supplement to traditional nutrition education ${ }^{(34,35)}$. Some evidence exists to support this approach, as prior research has shown that cooking skills education through cooking classes and engagement in food preparation activities in adolescence can lead to more healthful diet behaviours ${ }^{(12,36-38)}$. Additionally, efforts to increase cooking frequency should consider time constraints, lack of access to affordable, high-quality, fresh ingredients, as well as lack of cooking equipment, which limit the amount of food Americans are able to prepare themselves at home. Possible approaches to decreasing these common barriers to cooking include modifications to workplace cultures that allow for flexible schedules that would enable working parents to leave work in time to prepare dinner; or industry innovations to cut down on the time needed to cook a healthy dinner (e.g. wider distribution of pre-cut ingredients or selling all the components of a recipe together with a recipe card).

Although we found that roughly half of our nationally representative sample lives in a household where someone cooks dinner very frequently ( $\geq 5$ times) during the week, increasing cooking frequency may not be a reasonable behaviour modification for those who do not. Consistent with Virudachalam et al., our analysis of the associations between frequency of cooking dinner and sociodemographic characteristics suggests variation in cooking frequency by race, age, gender, education, household food security and whether or not one is born in the USA or another country. The finding that individuals with low socio-economic status cook a lot or very little (as compared with people with high socio-economic status who typically cook a moderate amount) suggests that the decision to cook may be 'forced' among lower-income and minority groups. In other words, cooking may be necessary (potentially due to financial constraints) or challenging (potentially due to time constraints) for poorer families ${ }^{(16)}$. Therefore, although home cooking is increasingly recognized as an obesity prevention measure and a means to consume a healthier diet, increasing cooking frequency may not be achievable for everyone. Therefore, other strategies are also needed to help infrequent cookers to better navigate the food environment outside the home. This might include individual-level interventions such as nutrition education emphasizing building food preparation skills and knowledge, as well as population-level interventions such as menu labelling on menu boards which were included the Patient Protection and Affordable Care $\mathrm{Act}^{(39)}$

The present study has several limitations. First, unmeasured food environment characteristics which are associated with consumption patterns, such as access to fresh fruits and vegetables, may bias these results towards the null. Second, due to limitations of the data (the NHANES does not code dietary recall data for whether or not a food was cooked at home), we were unable to examine consumption patterns or diet quality on days when cooking or not cooking, or based on whether or not specific items were cooked at home or not. However, by including measures of kilojoules consumed at home and away from home, as well as measures of frozen meals, meals not prepared from home per week and the number of ready-to-eat meals consumed in the past $30 \mathrm{~d}$, we attempt to mitigate this problem and provide a more nuanced description of consumption patterns and food preparation practices. Third, our measures of diet composition (overall energy intake, grams of protein, fibre, carbohydrates, fat and sugar) are not conclusive indicators of overall diet quality. On a population level, reducing the amount of fat, sugar, carbohydrates and energy intake are goals of the Dietary Guidelines for Americans ${ }^{(25)}$, but for any given individual changes in these measures may not result in improved diet quality. Fourth, our reliance on self-reported data for the single $24 \mathrm{~h}$ dietary recall and other diet quality measures may bias our results due to underreporting and unreliability resulting from recall and social desirability bias. For the same reasons, cooking frequency may be overestimated. Additionally, due to the way the food and beverage consumption data are converted into energy and nutrient intake information, there could be measurement error. Fifth, our reliance on cross-sectional data does not allow for causal inferences. Sixth, it is possible that cooking frequency categories are not mutually exclusive. To address this concern, and assess whether our cut-off points for this measure were meaningful, we replicated our analyses using multiple definitions of cooking frequency and found consistent results. Finally, our cooking measure only reports frequency of cooking dinner; the composition or consumption of the food that was cooked is unknowable, as is the frequency of cooking other meals. However, our results based on the measure of 'consumption of meals/ week not prepared from home' lend confidence to the assumption that frequency of cooking dinner is an appropriate proxy for overall cooking practices. Importantly, the definition of what it means to cook, and what constitutes dinner or a meal, is not standardized and could vary widely across individuals and populations ${ }^{(40)}$. Investigating how people interpret what food preparation activities and food items are included when respondents report having 'cooked' is an important area for future research. More research is also needed to understand how cooking is used as a weight-loss strategy for people who want to change their diets.

\section{Conclusion}

In conclusion, having someone in the household cook dinner more frequently is associated with lower consumption of total kilojoules, carbohydrates, fat, sugar and fast food. 
The benefits of cooking are present regardless of whether or not an individual is trying to lose weight. As we continue to search for solutions to the problem of obesity, it is important to consider mechanisms to reduce the barriers for adults among who might like to cook more as well as help adults who are unlikely to cook to more easily navigate the food environment outside the home.

\section{Acknowledgements}

Acknowledgements: The authors gratefully acknowledge the work of Seanna Vine, MPH in compiling the data set and providing feedback on the manuscript. Financial support: J.A.W. has no financial disclosures. S.N.B. was supported by the National Heart, Lung, and Blood Institute (grant number 1K01HL096409). No funder played any role in the design, analysis or writing of this article. Conflict of interest: None. Authorship: J.A.W. and S.N.B. conceived the study and developed the hypotheses. J.A.W. performed the statistical analysis. J.A.W. and S.N.B. contributed to the interpretation of study findings. J.A.W. drafted the manuscript and both authors contributed to the final draft. J.A.W. had full access to all of the data in the study and takes responsibility for the integrity of the data and the accuracy of the data analysis. Ethics of human subject participation: Ethical approval was not required.

\section{Supplementary material}

To view supplementary material for this article, please visit http://dx.doi.org/10.1017/S1368980014001943

\section{References}

1. Shao Q \& Chin KV (2011) Survey of American food trends and the growing obesity epidemic. Nutr Res Pract 5, 253-259.

2. McGuire S (2011) Todd, J.E., Mancino, L., Lin, B.-H. The impact of food away from home on adult diet quality. ERR-90, U.S. Department of Agriculture, Econ. Res. Serv., February 2010. Adv Nutr 2, 442-443.

3. Ayala GX, Rogers M, Arredondo EM et al. (2008) Awayfrom-home food intake and risk for obesity: examining the influence of context. Obesity (Silver Spring) 16, 1002-1008.

4. Powell LM, Nguyen BT \& Han E (2012) Energy intake from restaurants: demographics and socioeconomics, 2003-2008. Am J Prev Med 43, 498-504.

5. Drewnowski A \& Rehm CD (2013) Energy intakes of US children and adults by food purchase location and by specific food source. Nutr J 12, 59.

6. Flegal KM, Carroll MD, Ogden CL et al. (2010) Prevalence and trends in obesity among US adults, 1999-2008. JAMA 303, 235-241.

7. Cohen DA \& Bhatia R (2012) Nutrition standards for awayfrom-home foods in the USA. Obes Rev 13, 618-629.

8. Jabs J \& Devine CM (2006) Time scarcity and food choices: an overview. Appetite 47, 196-204.

9. Smith LP, Ng SW \& Popkin BM (2013) Trends in US home food preparation and consumption: analysis of national nutrition surveys and time use studies from 1965-1966 to 2007-2008. Nutr J 12, 45.
10. Zick CD \& Stevens RB (2010) Trends in Americans' food-related time use: 1975-2006. Public Health Nutr 13, 1064-1072.

11. Kolodinsky JM \& Goldstein AB (2011) Time use and food pattern influences on obesity. Obesity (Silver Spring) 19, $2327-2335$.

12. Lang T, Caraher M, Dixon P et al. (1999) Cooking Skills and Health. London: Health Education Authority.

13. Stead M, Caraher M, Wrieden W et al. (2004) Confident, fearful and hopeless cooks: findings from the development of a food-skills initiative. Br Food J 106, 274-287.

14. Cawley J \& Liu F (2012) Maternal employment and childhood obesity: a search for mechanisms in time use data. Econ Hum Biol 10, 352-364.

15. Devine CM, Farrell TJ, Blake CE et al. (2009) Work conditions and the food choice coping strategies of employed parents. J Nutr Educ Behav 41, 365-370.

16. Virudachalam S, Long JA, Harhay MO et al. (2014) Prevalence and patterns of cooking dinner at home in the USA: National Health and Nutrition Examination Survey (NHANES) 2007-2008. Public Health Nutr 17, 1022-1030.

17. Engler-Stringer R (2010) Food, cooking skills, and health: a literature review. Can J Diet Pract Res 71, 141-145.

18. Zick CD, Stevens RB \& Bryant WK (2011) Time use choices and healthy body weight: a multivariate analysis of data from the American Time Use Survey. Int J Behav Nutr Phys Act $\mathbf{8}, 84$.

19. Chenhall C (2010) Improving cooking and food preparation skills: a synthesis of the evidence to inform program and policy development. http://www.hc-sc.gc.ca/fn-an/nutrition/ child-enfant/cfps-acc-synthes-eng.php (accessed October 2013).

20. Weiss EC, Galuska DA, Khan LK et al. (2006) Weight-control practices among US adults, 2001-2002. Am J Prev Med 31, $18-24$.

21. Siu J, Giskes K \& Turrell G (2011) Socio-economic differences in weight-control behaviours and barriers to weight control. Public Health Nutr 14, 1768-1778.

22. Sacks FM, Bray GA, Carey VJ et al. (2009) Comparison of weight-loss diets with different compositions of fat, protein, and carbohydrates. New Engl J Med 360, 859-873.

23. Nestle M (2010) Strategies to prevent childhood obesity must extend beyond school environments. Am J Prev Med 39, 280-281.

24. Centers for Disease Control (2009) National Health and Nutrition Examination Survey. Hyattsville, MD: US Department of Health and Human Services, Centers for Disease Control and Prevention.

25. US Department of Agriculture \& US Department of Health and Human Services (2010) Dietary Guidelines for Americans, 2010, 7th ed. Washington, DC: US Government Printing Office.

26. World Health Organization (1988) Obesity: Preventing and Managing the Global Epidemic. Report of a WHO Consultation on Obesity. Geneva: WHO.

27. Bickel G, Nord M, Price C et al. (2000) Guide to Measuring Household Food Security (Revised 2000). Alexandria, VA: Office of Analysis, Nutriton, and Evaluation, Food and Nutrition Service, US Department of Agriculture.

28. Condrasky MD \& Hegler M (2010) How culinary nutrition can save the health of a nation. J Extension 48, issue 2, 2COM1; available at http://www.joe.org/joe/2010april/comm1. php.

29. Mancino L \& Gregory CA (2012) Does more cooking mean better eating? Estimating the relationship between time spent in food preparation and diet quality. Presented at 2012 Agricultural and Applied Economics Association Annual Meeting. Seattle, WA, USA, 12-14 August 2012. 
30. Hamrick KS, Andrews M, Guthrie J et al. (2011) How Much Time Do Americans Spend on Food? Economic Information Bulletin no. EIB-88. Washington, DC: US Department of Agriculture, Economic Research Service.

31. Cunningham-Sabo L \& Simons A (2012) Home economics: an old-fashioned answer to a modern-day dilemma? Nutr Today 47, 128-132.

32. Lichtenstein AH \& Ludwig DS (2010) Bring back home economics education. JAMA 303, 1857-1858.

33. Peregrin T (2010) Home economics makes a comeback: opportunities for RDs to become part of the curriculum. $J$ Am Diet Assoc 110, 1626, 1628-1629.

34. Condrasky MD, Griffin SG, Catalano PM et al. (2010) A formative evaluation of the Cooking with a Chef program. $J$ Extension 48, issue 2, 2FEA1; available at http://www.joe. org/joe/2010april/a1.php.
35. Caraher M, Seeley A, Wu M et al. (2013) When chefs adopt a school? An evaluation of a cooking intervention in English primary schools. Appetite 62, 50-59.

36. Levy J \& Auld G (2004) Cooking classes outperform cooking demonstrations for college sophomores. J Nutr Educ Behav 36, 197-203.

37. Caraher M, Dixon P, Lang T et al. (1999) The state of cooking in England: the relationship of cooking skills to food choices. Br Food J 101, 590-607.

38. Larson NI, Perry CL, Story M et al. (2006) Food preparation by young adults is associated with better diet quality. $J \mathrm{Am}$ Diet Assoc 106, 2001-2007.

39. Patient Protection and Affordable Care Act (2010) Public Law 111-148, Mar. 23, 2010.

40. Short F (2006) Kitchen Secrets: The Meaning of Cooking in Everyday Life. Oxford: Berg.

\section{Appendix}

Question wording for diet quality measures in the National Health and Nutrition Examination Survey (NHANES)

\begin{tabular}{|c|c|}
\hline Measure & Question wording in NHANES \\
\hline Meals not prepared at home & $\begin{array}{l}\text { 'How many meals did you get that were prepared away from home in places such as restaurants, fast } \\
\text { food places, food stands, grocery stores, or from vending machines?' }\end{array}$ \\
\hline Ready-to-eat meals & 'During the past 30 days, how often did you eat "ready to eat" foods from the grocery store?' \\
\hline Frozen meals & 'During the past 30 days, how often did you eat frozen meals or frozen pizzas?' \\
\hline
\end{tabular}

